\title{
PTEN sensitizes epidermal growth factor-mediated proliferation in endometrial carcinoma cells
}

\author{
LIU LIN TANG ${ }^{1}$, YASUHIRO YOKOYAMA ${ }^{1}$, XIAOYUN WAN ${ }^{2}$, \\ SHIGENORI IWAGAKI ${ }^{1}$, KENJI NIWA ${ }^{1}$ and TERUHIKO TAMAYA ${ }^{1}$ \\ ${ }^{1}$ Department of Obstetrics and Gynecology, Gifu University School of Medicine, 1-1 Yanagido, Gifu 501-1194, \\ Japan; ${ }^{2}$ Zhejiang Woman's Hospital, Medical School of Zhejiang University, Hangzhou, Zhejiang, P.R. China
}

Received April 22, 2005; Accepted June 20, 2005

\begin{abstract}
The fact that the genetic alterations of PTEN are frequently found in hormone-dependent cancers, such as endometrial, breast, and prostate cancers, might suggest the involvement of PTEN in the hormone-dependent cell growth of such tumors. Estrogen promotes the cell growth of the tumors by inducing peptide growth factors in part. We analyzed the possible involvement of PTEN in peptidegrowth factor-dependent cell growth in endometrial carcinoma cells. PTEN-null Ishikawa cells were efficiently infected with recombinant adenovirus at 20 MOI (multiplicity of infection) to express PTEN protein. In PTEN-IK cells, phospho-Akt/PKB was down-regulated regardless of the consistent expression of Akt/PKB. The cell growth of parental IK cells was significantly stimulated by EGF and IGF-I, and PTEN-IK cells were further sensitized to the EGF-or IGF-I-growth stimulation. EGFR antibody could completely compromise the stimulatory effects of EGF in both cell lines. Wortmannin, a PI3K inhibitor, or UO126, a MAPK inhibitor, partly suppressed EGF-mediated cell growth stimulation in both cell lines. EGF augmented the level of phospho-Akt/PKB of PTEN-IK cells more effectively than that of parental IK cells. These results imply that the dysfunction of PTEN leads cells into a less-sensitive phenotype to peptide growth factors by constitutive activation of the $\mathrm{PI} 3 \mathrm{~K} / \mathrm{Akt} / \mathrm{PKB}$ signaling pathway in endometrial carcinoma.
\end{abstract}

\section{Introduction}

PTEN functions as a lipid phosphatase to regulate phosphatidylinositol 3,4,5-trisphosphate (PIP3) as well as a tyrosine phosphatase to down-modulate the signaling pathways that involve focal adhesion kinase (FAK) or Shc. PTEN regulates

Correspondence to: Dr Liu Lin Tang, Department of Obstetrics and Gynecology, Gifu University School of Medicine, 1-1 Yanagido, Gifu 501-1194, Japan

E-mail: tangliulin@hotmail.com

Key words: PTEN, endometrial carcinoma, EGFR, EGF, PI3K, MAPK many normal cell processes, including growth, adhesion, migration, invasion and apoptosis (1). Genetic alterations of PTEN are observed in a variety of cancers, though it is frequently found in sex steroid hormone-associated cancers such as endometrial, prostate, and breast cancers (2). During the development of such tumors, most of the tumors lose the sex steroid hormone-dependency. This fact suggests that PTEN might modulate the action of sex steroid hormones and that the dysfunction of PTEN is involved in the hormoneindependent cell growth of such tumors.

The endometrium is a major target tissue of estrogen. Persistent and excessive estrogen exposure is considered to induce a series of endometrial neoplasms. The estrogen/ estrogen receptor (ER) complex switches on a variety of gene transcriptions. Some peptide growth factors, including EGF, TGF $\alpha$ and IGF-I, would be regulated by estrogens $(3,4)$ but other peptide growth factors, including HGF and FGF, might be produced indirectly by sex steroid hormones (5). These peptide growth factors stimulate the growth of endometrial carcinoma cells $(6,7)$. EGF, TGF $\alpha$ and IGF-I are expressed in the endometrial epithelial cells and their expression levels vary with the stage of the menstrual cycle $(8,9)$. On the contrary, endometrial carcinoma reduces the expressions of these peptide growth factors $(10,11)$. Considering the fact that most endometrial neoplasia lose estrogen-dependency during the progression of histological grade, the reduced expression of the peptide growth factor in endometrial carcinoma might be a result of the acquisition of sex steroid hormone-independency.

Upon activation by the binding of EGF or other ligands, the EGF-receptor (EGFR) undergoes dimerization and autophosphorylation, leading to the stimulation of many different signal transduction cascades, such as the mitogenactivated protein kinase (MAPK) pathway, phosphoinositide 3-kinases (PI3K), Akt/PKB, and several transcriptional regulators (12). PTEN, on the other hand, antagonizes the action of PI3K by dephosphorylating PIP3. Thus, the responsiveness of EGF or other peptide growth factors of cells may be affected by the PTEN protein. In endometrial carcinomas, approximately half of the cases possess some deficiency in the PTEN gene $(13,14)$. The dysfunction of the PTEN protein could be associated with the development of endometrial carcinomas although its involvement in the responsiveness to peptide growth factors is unknown. In the 
present study, we analyzed the possible involvement of PTEN in growth factor responsiveness, especially EGF responsiveness, in an endometrial carcinoma cell.

\section{Materials and methods}

Cell. Ishikawa cells, an endometrial carcinoma cell line, were used in this study. This cell does not express the PTEN protein due to missense mutations (15). The cells were maintained in modified Eagle's medium (MEM) supplemented with $10 \%$ fetal bovine serum under an atmosphere of $95 \%$ air and $5 \%$ $\mathrm{CO}_{2}$. HEK293 cells were maintained in alpha-modified MEM supplemented with $10 \%$ FBS.

Recombinant adenovirus preparation. To produce a recombinant adenoviral vector, the Adeno- $\mathrm{X}^{\mathrm{TM}}$ expression system (Clontech Laboratories Ltd., Tokyo, Japan) was used. In brief, a wild-type PTEN cDNA of approximately $1.3 \mathrm{~K}$ base-pairs was excised from pcDNA3-PTEN (15) with SpeI and ApaI restriction enzymes and subcloned into NheI and ApaI sites of the pShuttlr vector (designated as pShuttlePTEN). Approximately a $2.6 \mathrm{Kbp}$ expression cassette from the pShuttle-PTEN vector was subcloned into the I-CeuI and PL-SceI sites of pAdeno-X plasmid. The plasmid DNA was digested with PacI restriction enzyme before transfection into HEK293 cells. Viruses were propagated in the cells and extracted by three consecutive freeze-thaw cycles of the cells. Viral titer was measured in a limiting-dilution bioassay using the HEK 293 cells.

The recombinant adenovirus carrying LacZ gene was produced by the same procedure with a pShuttle-LacZ plasmid (Clontech Laboratories Ltd.,Tokyo, Japan). Of the Ishikawa cells, the subline in which the PTEN protein was efficiently induced by the recombinant adenovirus was designated as PTEN-IK cells. Lac-IK cells were designated as the cells infected at the same MOI as PTEN-IK cells with a recombinant adenovirus carrying the LacZ gene.

Effect of growth factors on the cell growth and modification of signal transduction inhibitor. Recombinant human EGF and recombinant human IGF-I were purchased from Invitrogen Co., Ltd., (Tokyo, Japan). Recombinant human HGF and recombinant basic FGF were purchased from Genzyme Techne (Minneapolis, MN). Wortmannin and UO126 were purchased from Upstate (Charlottesville, VA). A mouse monoclonal EGFR antibody (clone 528) was purchased from Santa Cruz Biotechnology (Santa Cruz, CA).

Approximately $5 \times 10^{4}$ cells were seeded in 24 -well culture plates on the 5 th day after infection. At $24 \mathrm{~h}$, various concentrations of growth factors were added to the serumfree medium. At $72 \mathrm{~h}$, MTT $(180 \mu \mathrm{g})$ was added to each well. The culture medium was replaced with $1 \mathrm{ml}$ dimethyl sulfoxide. After $10 \mathrm{~min}$ of agitation, an absorption of $540 \mathrm{~nm}$ was read using an Ultospec $200 \mathrm{UV} /$ Visible spectrophotometer (Pharmacia Biotech, Inc., Uppsala, Sweden).

Western blot. Cells were lyzed in cell lysis buffer [10 $\mathrm{mM}$ Tris-Hcl, $\mathrm{pH} 7.4,150 \mathrm{mM} \mathrm{NaCl}, 0.1 \%$ polyoxyethylene (10) octylphenyl ether (triton X-100), $10 \mathrm{mM}$ 2-mercaptoethanol, aprotinin $2 \mu \mathrm{g} / \mathrm{ml}$, leupeptin $5 \mu \mathrm{g} / \mathrm{ml}$ ] by a Sonifier 250
A

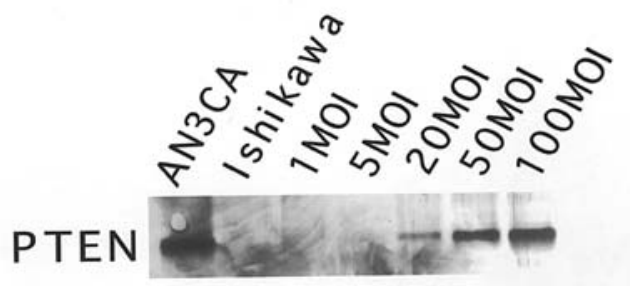

B

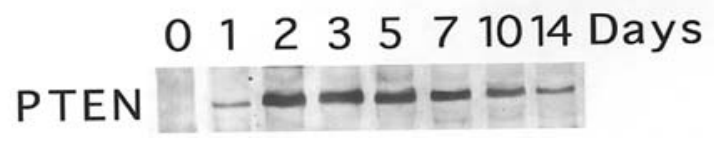

Figure 1. Adenovirus-mediated PTEN induction in Ishikawa cells. A, expression of PTEN protein at various multiplicities of infection. B, time course of PTEN protein expression at $20 \mathrm{MOI}$. MOI, multiplicity of infection. AN3CA, endometrial carcinoma cells as a positive control.

(Branson Sonic Power Company, Danbury, CT). The lysates were centrifuged at $10000 \mathrm{~g}$ for $30 \mathrm{~min}$ and the supernatant was recovered. The boiled lysate $(10 \mu \mathrm{g})$ was subjected to 4-20\% SDS-PAGE under reducing conditions and blotted onto nitrocellulose membrane. The blot was probed with a mouse monoclonal antibody against PTEN (A2B1, Santa Cruz Biotech), rabbit polyclonal antibody against Akt (New England Biolabs, Inc.), rabbit polyclonal antibody against phospho-Akt (New England Biolabs, Inc.) and rabbit polyclonal antibody against ERK1 (k-23, Santa Cruz Biotech). Immune complexes were identified and visualized by the ECL Western blotting detection system (Amersham Pharmacia Biotech, Tokyo, Japan).

To quantify the relative amount of phospho-Akt/PKB in the cells, the density of their bands was measured by BioImage (Millipore Corp., Bedford, MA).

$R T-P C R$. EGF, Neu and EGFR expressions were analyzed by RT-PCR. Total RNA was extracted by Isogene Reagents (NipponGene, Inc., Toyama, Japan) in accordance with the manufacturer's procedure. Total RNA $(5 \mu \mathrm{g})$ was reverse transcribed into cDNA with random hexadeoxynucleotides and murine reverse transcriptase from First-Strand cDNA Synthesis Kit (Pharmacia-Biotech, Inc., Tokyo, Japan).

PCR was performed with the following sets: EGF, 5'-CT CAGGGAAGATGACCACCA and 5'-GCAGACAGCCACC ACGATGA; EGFR, 5'-CACAGGAC GGGGACCAGACAA and 5'-CCACCACCAGCAGCAAGAGGA; and Neu, 5'-GA TGTATTTGATGGTGACCT and 5'-ATCTGGCTGGTTCA CATATT.

The conditions of the PCR were $94^{\circ} \mathrm{C}$ for $1 \mathrm{~min}, 55^{\circ} \mathrm{C}$ for $1 \mathrm{~min}$, and $72^{\circ} \mathrm{C}$ for $1 \mathrm{~min}$ for 28 cycles.

\section{Results}

PTEN induction by recombinant adenovirus. The recombinant adenovirus efficiently induced PTEN protein in Ishikawa cells. The Ishikawa cells required 20 MOI to induce PTEN protein expression. The expression level of PTEN was dose-dependent, although a higher concentration of virus induced cell apoptosis. 


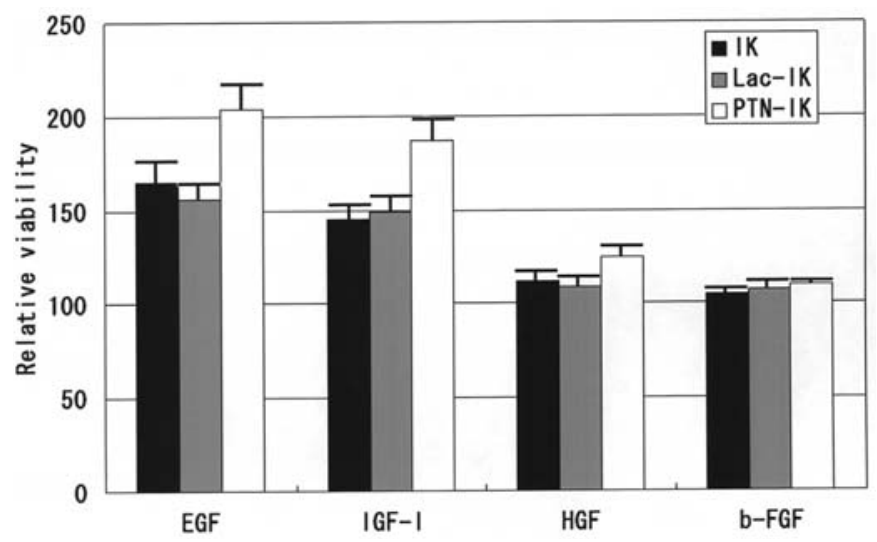

Figure 2. Effects of growth factors on the cell viability. The effects of growth factors on the cell viability were analyzed by MTT assay. Cell viability under the influence of the peptide growth factors $(10 \mathrm{ng} / \mathrm{ml})$ is expressed as percent ratio to that of the untreated control. EGF or IGF-I significantly augmented the cell growth of IK cells. They further augmented the cell growth of PTEN-IK cells as compared to that of IK cells. HGF and ßFGF exert a minimal effect on the cell growth.

When the cells were infected at $20 \mathrm{MOI}$, the protein expression was detected as early as $18 \mathrm{~h}$ after infection and reached maximal expression at $48 \mathrm{~h}$. This expression level lasted for several days (Fig. 1). At this multiplicity of infection, cells (PTEN-IK cells) survived for over 2 weeks with a steady expression of PTEN.

Effects of peptide growth factors on the cell growth. The effects of peptide growth factors on the cell growth were analyzed by MTT assay. EGF and IGF-I at $1 \mathrm{ng} / \mathrm{ml}$ to $100 \mathrm{ng} /$ $\mathrm{ml}$ significantly augmented the cell growth of parental IK cells, whereas HGF and B-FGF exerted a minimal effect on cell growth. When IK cells were exposed to $10 \mathrm{ng} / \mathrm{ml}$ of EGF or IGF-I, the cell viability was 165 or $143 \%$, respectively, compared to the untreated control (Fig. 2). In PTEN-IK cells, the growth stimulatory effect of EGF or IGF-I was further augmented into 202 or $185 \%$, respectively. HGF augmented cell growth in PTEN-IK cells to some extent.

Effect of PTEN on gene expression. IK cells expressed EGF, EGFR and Neu mRNAs. Of PTEN-IK cells, the endogenous EGF was up-regulated, and the levels of EGFR and Neu mRNAs were equivalent to those of IK cells (Fig. 3A). IK cells expressed the steady states of phospho-Akt/PKB protein, although the phospho-Akt/PKB protein was down-regulated in PTEN-IK cells regardless of the consistent level of the Akt/ PKB protein. Of the PTEN-IK cells, however, the expression levels of endogenous ERK1 and ERK 2 were consistent with those of the parental IK cells ( Fig. 3B).

Effects of inhibitors on EGF-mediated cell growth stimulation. We analyzed the effect of antibody against EGFR, PI3K inhibitor or MAPK inhibitor on EGF-mediated growth stimulation (Fig. 4). The growth stimulatory effect of EGF $(10 \mathrm{ng} / \mathrm{ml})$ was completely blocked by the antibody, suggesting that EGFR and the downstream signal network could be associated with growth stimulation. Next, the effects of PI3K inhibitor or MAPK inhibitor on EGF-mediated growth
A

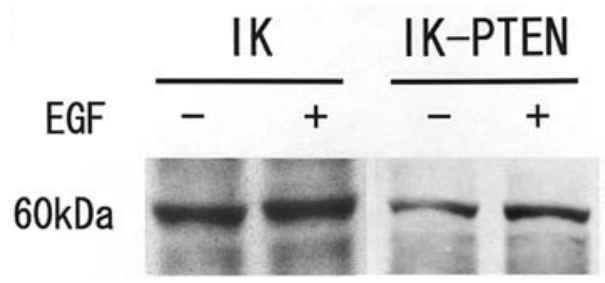

B

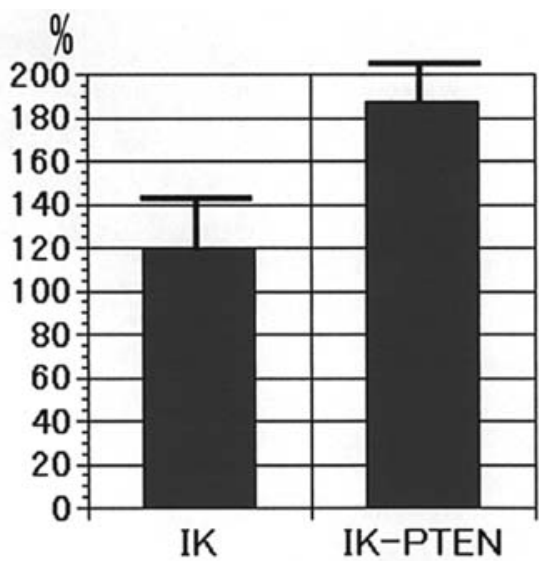

Figure 3. Expression levels of EGF, EGFR and HER-2 mRNAs (A), and Akt/PKB, phospho-AKT/PKB, ERK1 and ERK2 proteins (B). EGF is upregulated in the PTEN-IK cells, while phospho-Akt/PKB was down-regulated. Adenovirus-mediated gene transduction of PTEN does not modulate the expression of the other molecules. Lane 1, IK cells; lane 2, Lac-IK cells; lane 3, PTEN-IK cells.

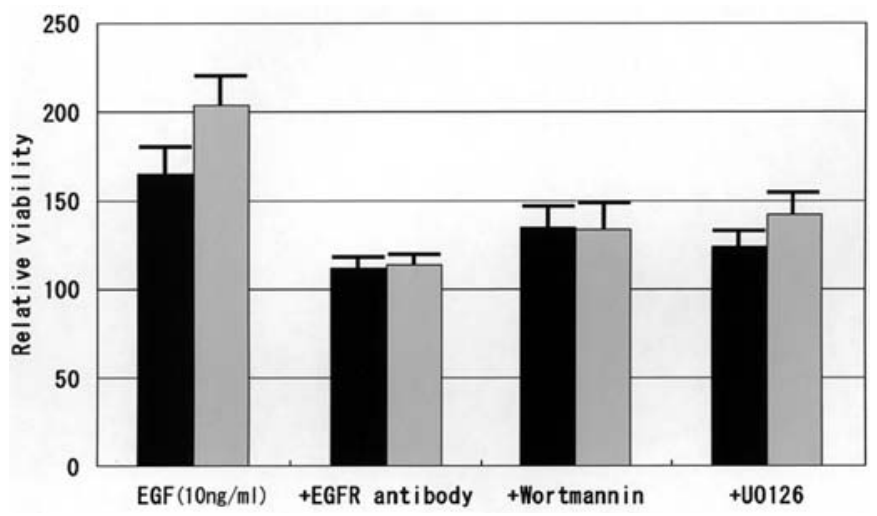

Figure 4. Effects of the EGFR antibody, wortmannin or UO126 on the cellgrowth-stimulation of EGF. Cell viability was assessed by MTT assay, and relative cell viability was expressed as percent ratio to the untreated control. The antibody against EGFR almost completely blocks the stimulation. UO126 or wortmannin inhibits it in part.

stimulation were analyzed. Neither wortmannin $(1 \mu \mathrm{M})$, a PI3K inhibitor, nor UA-126 $(10 \mu \mathrm{M})$, a MAPK inhibitor, affected the growth of IK cells. At this concentration of wortmaninn, the growth stimulatory effect of EGF $(10 \mathrm{ng})$ was down-regulated from 201 to $134 \%$ in PTEN-IK cells. In IK cells, it was reduced from 165 to $134 \%$. Wortmaninn inhibited the EGF-mediated growth stimulation of PTEN-IK cells more efficiently than that of IK cells. UA126 $(10 \mu \mathrm{M})$ also showed inhibitory effects on the EGF-stimulation of both cells.

Next, we analyzed the effects of EGF on the phosphoAkt/PKB. EGF augmented the level of phospho-Akt/PKB to 


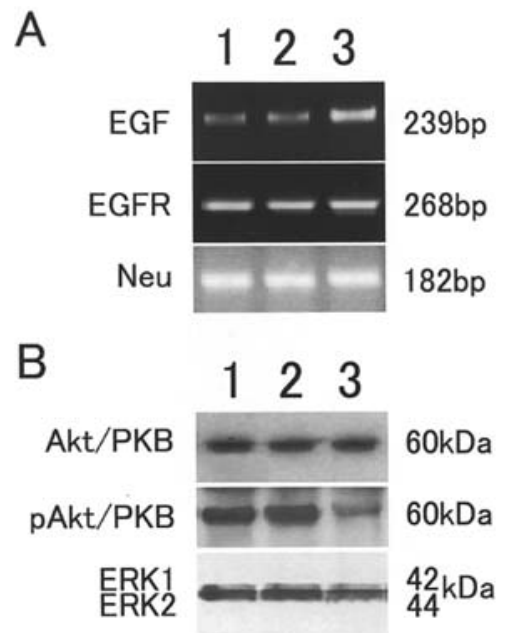

Figure 5. Effect of EGF on the level of phospho-Akt/PKB. The expression levels of phospho-Akt/PKB were analyzed by western blot (A) and densitometry analysis (B). The signal intensity of phospho-Akt/PKB in the cells treated with EGF is expressed as relative percentage of the untreated control. The signal intensity of phospho-Akt/PKB is significantly increased in PTEN-IK cells $(\mathrm{p}<0.01)$.

$185 \%$ in PTEN-IK cells, whereas it exerted minimal effects on phospho-Akt/PKB in parental IK cells (Fig. 5).

\section{Discussion}

In the endometrium, EGF is expressed in epithelial cells and its level is increased at the late follicular and luteal stages, compared to the early follicular stage (8). The increment in the expression level of EGF is compatible with the level of plasma estrogen, so this cyclic change in EGF expression in the endometrium could be a result of estrogen action. The EGFR is expressed in the normal endometrium throughout the menstrual cycle (16). On the other hand, EGF expression is observed in limited cases of endometrial carcinoma (11), whereas the expression of EGFR is consistently frequent in a series of endometrial neoplasias (17). These observations might suggest that EGF regulates the cell growth of normal endometrium in an estrogen-dependent manner and that this regulatory system is down-regulated in most endometrial carcinomas by the loss of estrogen dependency. In the present study, we observed the augmentated expression of endogenous EGF in PTEN-IK cells when compared with parental IK cells. This implies that PTEN itself regulates EGF expression. Infrequent expression of EGF in endometrial carcinomas can be explained partly by PTEN dysfunction. We have found that IK cells express EGF mRNA, but the EGF protein has been demonstrated to be below the assay level in the conditioned medium of IK cells (6). We evaluated the level of EGF in the culture medium of PTEN-IK cells but did not find its presence. Regardless of the augmentation of EGF mRNA in PTEN-IK cells, the level of EGF protein would be extremely low or negligible.

The ligation of EGF to an extracellular ligand-binding domain of EGFR induces receptor dimerization. The dimerization of EGFR is required for cross-phosphorylation of the receptors and subsequent formation of docking sites for signaling complexes composed of cytoplasmic enzymes and adaptor proteins (12). EGFR (HER-1) molecules dimerize each other, but do with other EGFR members such as Neu (HER-2), ErbB-3 (HER-3) or ErbB-4 (HER-4). The major partner of EGFR is Neu, though Neu does not bind ligands, even in a heterodimeric receptor complex (18). The dimer of EGFR-Neu is more stable at the cell surface than the other dimers and can decrease the rate of ligand dissociation from the cognate receptor, EGFR. In the present study, we observed that expression of Neu mRNA was not affected by PTEN induction. In addition, it has been reported that EGF reduces Neu expression in ovarian carcinoma cells (19). We analyzed the effects of exogenous EGF on the expression levels of EGFR and Neu but did not find any alterations in IK cells and PTEN-IK cells. Therefore, augmented sensitivity against EGF in PTEN-IK cells could be the result of alteration in the downstream signaling networks. The observation that the EGFR antibody completely blocked EGF-mediated growth stimulation in both cells, supports this hypothesis. However, the network is very complex. Key molecules of the downstream signaling pathways include MAPK, PKB, PI3K, and several transcriptional regulators.

Wortmannin partly blocked EGF-mediated growth stimulation in both cells but did so more effectively in PTEN-IK cells than in IK cells. In PTEN-IK cells, induced PTEN protein would down-regulate cellular PIP3 concentration, In such a state, the inhibition of PI3K by wortmannin would further down-regulate PIP3. As a result, growth inhibition by wortmanin is more prominent in PTENIK cells than in parental IK cells. Similarly, UO-126 blocked it partly in both cells. These observations imply that MAPK and PI3K are activated by EGF in both cells.

In PTEN-IK cells, the level of phospho-Akt/PKB was down-regulated. EGF augmented the level of phospho-Akt/ PKB of PTEN-IK cells more significantly than that of IK cells. This suggests that exogenous EGF stimulates the Akt/ PKB of PTEN-IK cells more potently than that of parental IK cells. IK cells allow constitutive activation of PIP3 due to lack of functional PTEN protein. In the IK cells, the downstream signaling pathway including $\mathrm{Akt} / \mathrm{PKB}$ is constitutively activated. On the other hand, PIP3 would be constitutively converted into PIP2 in PTEN-IK cells. PIP2 would be in turn recycled to participate in the signal transduction of EGF. This phosphorylation and subsequent dephosphorylation cycle of inositol-phospholipids would be important in transmitting the signals through PI3K. Thus, we conclude that, by regulating the phosphorylation/dephosphorylation cycles between PIP2 and PIP3, PTEN is actively involved in the signal transduction of EGF in endometrial carcinoma cells.

\section{References}

1. Yamada KM and Araki M: Tumor suppressor PTEN: modulator of cell signaling, growth, migration and apoptosis. J Cell Sci 114: 2375-2382, 2001.

2. Li J, Yen C, Liaw D, Podsypanina K, Bose S, Wang SI, et al: PTEN, a putative protein tyrosine phosphatase gene mutated in human brain, breast, and prostate cancer. Science 275: 1943-1947, 1997.

3. Bigsby RM: Control of growth and differentiation of the endometrium: the role of tissue interactions. Ann NY Acad Sci 955: 110-117, 2002.

4. Murphy LJ: Growth factors and steroid hormone action in endometrial cancer. J Steroid Biochem Mol Biol 48: 419-423, 1994. 
5. Spencer TE and Bazer FW: Biology of progesterone action during pregnancy recognition and maintenance of pregnancy. Front Biosci 7: d1879-d1898, 2002.

6. Reynolds RK, Hu C and Baker VV: Transforming growth factoralpha and insulin-like growth factor-I, but not epidermal growth factor, elicit autocrine stimulation of mitogenesis in endometrial cancer cell lines. Gynecol Oncol 70: 202-209, 1998.

7. Pearl ML, Talavera F, Gretz HF III, Roberts JA and Menon KM: Mitogenic activity of growth factors in the human endometrial adenocarcinoma cell lines HEC-1-A and KLE. Gynecol Oncol 49: 325-332, 1993.

8. Imai T, Kurachi H, Adachi K, Adachi H, Yoshimoto Y, Homma H, et al: Changes in epidermal growth factor receptor and the levels of its ligands during menstrual cycle in human endometrium. Biol Reprod 52: 928-938, 1995.

9. Tang XM, Rossi MJ, Masterson BJ and Chegini N: Insulin-like growth factor I (IGF-I), IGF-I receptors, and IGF binding proteins 1-4 in human uterine tissue: tissue localization and IGF-I action in endometrial stromal and myometrial smooth muscle cells in vitro. Biol Reprod 50: 1113-1125, 1994.

10. Maiorano E, Loverro G, Viale G, Giannini T, Napoli A and Perlino E: Insulin-like growth factor-I expression in normal and diseased endometrium. Int J Cancer 80: 188-193, 1999.

11. Yokoyama Y, Takahashi Y, Hashimoto M, Morishita S and Tamaya T: Immunohistochemical study of estradiol, epidermal growth factor, transforming growth factor alpha and epidermal growth factor receptor in endometrial neoplasia. Jpn J Clin Oncol 26: 411-416, 1996.

12. Yarden Y: The EGFR family and its ligands in human cancer, signalling mechanisms and therapeutic opportunities. Eur J Cancer 37 (Suppl 4): S3-S8, 2001.
13. Kong D, Suzuki A, Zou TT, Sakurada A, Kemp LW, Wakatsuki S, et al: PTEN1 is frequently mutated in primary endometrial carcinomas. Nat Genet 17: 143-144, 1997.

14. Tashiro H, Blazes MS, Wu R, Cho KR, Bose S, Wang SI, et al: Mutations in PTEN are frequent in endometrial carcinoma but rare in other common gynecological malignancies. Cancer Res 57: 3935-3940, 1997.

15. Wan X, Yokoyama Y, Shinohara A, Takahashi Y and Tamaya T: PTEN augments staurosporine-induced apoptosis in PTEN-null Ishikawa cells by downregulating PI3K/Akt signaling pathway. Cell Death Differ 9: 414-420, 2002.

16. Prentice A, Thomas EJ, Weddell A, McGill A, Randall BJ and Horne $\mathrm{CH}$ : Epidermal growth factor receptor expression in normal endometrium and endometriosis: an immunohistochemical study. Br J Obstet Gynaecol 99: 395-398, 1992.

17. Nyholm HC, Nielsen AL and Ottesen B: Expression of epidermal growth factor receptors in human endometrial carcinoma. Int $\mathbf{J}$ Gynecol Pathol 12: 241-245, 1993.

18. Tzahar E, Waterman H, Chen X, Levkowitz G, Karunagaran D, Lavi S, et al: A hierarchical network of interreceptor interactions determines signal transduction by Neu differentiation factor/ neuregulin and epidermal growth factor. Mol Cell Biol 16: 5276-5287, 1996.

19. Marth C, Lang T, Cronauer MV, Doppler W, Zeimet AG, Bachmair F, et al: Epidermal growth factor reduces HER-2 protein level in human ovarian carcinoma cells. Int J Cancer 52: 311-316, 1992. 